\title{
Nature and origins of mathematics difficulties in very preterm children: a different etiology than developmental dyscalculia
}

\author{
Victoria Simms ${ }^{1,2}$, Camilla Gilmore ${ }^{3}$, Lucy Cragg ${ }^{4}$, Sarah Clayton ${ }^{3}$, Neil Marlow ${ }^{5}$ and Samantha Johnson ${ }^{1}$
}

BACKGROUND: Children born very preterm $(<32 w k)$ are at high risk for mathematics learning difficulties that are out of proportion to other academic and cognitive deficits. However, the etiology of mathematics difficulties in very preterm children is unknown. We sought to identify the nature and origins of preterm children's mathematics difficulties.

METHODS: One hundred and fifteen very preterm children aged 8-10 y were assessed in school with a control group of 77 term-born classmates. Achievement in mathematics, working memory, visuospatial processing, inhibition, and processing speed were assessed using standardized tests. Numerical representations and specific mathematics skills were assessed using experimental tests.

RESULTS: Very preterm children had significantly poorer mathematics achievement, working memory, and visuospatial skills than term-born controls. Although preterm children had poorer performance in specific mathematics skills, there was no evidence of imprecise numerical representations. Difficulties in mathematics were associated with deficits in visuospatial processing and working memory.

CONCLUSION: Mathematics difficulties in very preterm children are associated with deficits in working memory and visuospatial processing not numerical representations. Thus, very preterm children's mathematics difficulties are different in nature from those of children with developmental dyscalculia. Interventions targeting general cognitive problems, rather than numerical representations, may improve very preterm children's mathematics achievement.

C hildren born very preterm ( $<32 \mathrm{wk}$ gestation) are at high risk for adverse cognitive outcomes including intellectual disability, learning difficulties, and special educational needs $(1,2)$. In particular, very preterm children have substantial difficulties with mathematics that are not accounted for by low intelligence (IQ) (see ref. (3) for review). Although mathematics difficulties are especially common, the nature and causes of these difficulties remain unexplained. To elucidate underlying mechanisms, it is necessary to consider the influence of both general cognitive skills and specific mathematics skills.
A range of executive functions have been found to be important for mathematical achievement (4), including working memory (5), inhibition (6), visuospatial skills (7), and processing speed (8). Alongside these, a number of specific mathematics processes are also related to achievement (9), including basic counting skills (10), the ability to apply efficient strategies (11), the accuracy and speed of retrieval of basic number facts (12), and the conceptual understanding of mathematics (9).

Most recently, studies have identified the importance of the approximate number system, a cognitive system that supports the representation and manipulation of quantity information, for mathematical achievement (13). The acuity of approximate number system representations are measured using magnitude comparison tasks, which require participants to quickly compare two quantities presented in either a symbolic or nonsymbolic format (Figure 1 (6)). Measures of accuracy and reaction time (RT) on these tasks have been shown to correlate with mathematics achievement (13). In particular, individuals with developmental dyscalculia, a learning disorder characterized by specific difficulties in mathematics, have poorer approximate number system acuity compared to those with typical development (14).

While progress has been made in understanding the cognitive mechanisms underlying developmental dyscalculia, researchers have yet to undertake a comprehensive evaluation of the general cognitive functions and mathematics specific processes which may underpin mathematics difficulties in preterm children. Although the similar behavioral profiles suggest that the same mechanisms may underlie difficulties in both groups, there is reason to believe that their cognitive profile may differ. It is plausible that very preterm children's difficulties may stem from their core deficits in visuospatial processing, working memory, and processing speed rather than imprecise numerical representations (3). A handful of studies have assessed numerical representations in very preterm children with contradictory results (15-18). Where imprecise numerical representations have been reported $(17,18)$, the experimental techniques used were unconventional and increased the general cognitive demands of the task making them especially detrimental for preterm

\footnotetext{
'Department of Health Sciences, University of Leicester, Leicester, UK; ${ }^{2}$ School of Psychology, Ulster University, Coleraine, UK; ${ }^{3}$ Mathematics Education Centre, Loughborough University, Loughborough, UK; ${ }^{4}$ School of Psychology, University of Nottingham, Nottingham, UK; ${ }^{5}$ Department of Academic Neonatology, Institute for Women's Health, University College London, London, UK. Correspondence: Samantha Johnson (sjj19@le.ac.uk) 
a

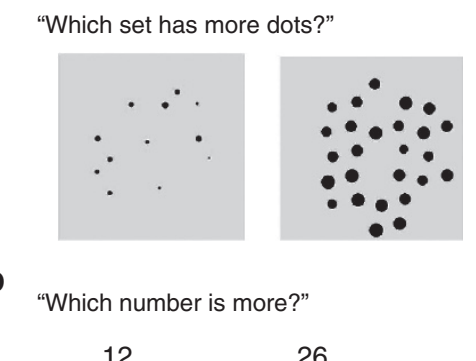

12

26

Figure 1. Recent research has highlighted that the precision of an individual's underlying numerical representations can predict current and future mathematics achievement. Experimental methods have been developed to explore various characteristics of these representations. Nonsymbolic magnitude comparison tasks (a), in which participants are required to select the more numerous of two arrays of dots, give a measure of the precision of nonsymbolic representations within the approximate number system. Symbolic magnitude comparison tasks (b), in which participants rapidly select the larger of two digits, index the accuracy of mapping between nonsymbolic and symbolic numerical representations.

children, or mathematical achievement was not assessed (17). Thus, it is difficult to ascertain whether group differences were methodological artifacts or were related to poor achievement.

We have previously observed that basic quantity processing explained a small amount of unique variance $(2 \%)$ in the mathematical achievement of extremely preterm $(<26 \mathrm{wk})$ children. However, as general cognitive deficits accounted for the majority of variance, this suggests that these may play an important role and that there may be an interaction between the two skill domains (19). Crucially, as no studies have measured general cognitive skills concurrently with numerical representations and a range of specific mathematics skills (18), the cognitive bases of preterm children's mathematical difficulties remain unknown. The aims of the present study were to determine whether mathematical processing deficits in very preterm children are similar to those of children with developmental dyscalculia and to identify the specific nature of mathematics difficulties in children born very preterm.

\section{RESULTS}

There were no significant differences between very preterm children recruited $(n=117)$ and not recruited $(n=149)$ in birthweight (mean difference $-0.43 \mathrm{~g}$; $95 \% \mathrm{CI}-101.36,100.50, P$ $=0.99)$ or gestational age $(-0.17 \mathrm{wk},-0.70,0.37, P=0.54)$, and there was no association between recruitment and IMD group $\left(\chi^{2}=0.31 ; P=0.86\right)$, demonstrating that the very preterm sample were representative of the total eligible population. In addition, very preterm and control participants were successfully matched for sex, age, mother's age, maternal educational qualification, and occupational class. However, there was a significant association between group and area-level socioeconomic deprivation as measured by the IMD tertile score (20) (Table 1). There were no group differences between very preterm children who did or did not have a matched control in nonverbal IQ $\left(t_{111}=-0.25 ; P=0.247\right)$ or mathematics achievement $\left(t_{111}=-0.97 ; P=0.330\right)$.
Table 1. Sample characteristics

\begin{tabular}{|c|c|c|c|}
\hline Characteristic & Controls, $n=77$ & Very preterm, $n=115$ & $P$ \\
\hline $\begin{array}{l}\text { Gestational age (weeks), } \\
\text { median (IQR) }\end{array}$ & - & $28.57(2.01)$ & - \\
\hline Birthweight (g), mean (SD) & - & $1,213.20(365.41)$ & - \\
\hline Male sex, $n(\%)$ & 40 (51.9\%) & $63(54.8 \%)$ & 0.70 \\
\hline $\begin{array}{l}\text { Age at assessment (years), } \\
\text { mean (SD) }\end{array}$ & $9.51(0.68)$ & $9.70(0.69)$ & 0.08 \\
\hline $\begin{array}{l}\text { Mother's age (years), mean } \\
\text { (SD) }\end{array}$ & $40.18(5.96)$ & $40.32(5.47)$ & 0.89 \\
\hline $\begin{array}{l}\text { Index of multiple } \\
\text { deprivation tertile: }\end{array}$ & & & 0.048 \\
\hline High deprivation & $37(49.3 \%)$ & $43(37.4 \%)$ & \\
\hline Middle deprivation & $18(24.0 \%)$ & $21(18.3 \%)$ & \\
\hline Low deprivation & $20(26.7 \%)$ & $51(44.3 \%)$ & \\
\hline Socio-occupational class: & & & 0.51 \\
\hline $\begin{array}{l}\text { Professional and } \\
\text { managerial }\end{array}$ & $25(32.1 \%)$ & $40(33.6 \%)$ & \\
\hline $\begin{array}{l}\text { Intermediate and } \\
\text { technical }\end{array}$ & $28(35.9 \%)$ & $30(25.2 \%)$ & \\
\hline Routine and semiroutine & $6(7.7 \%)$ & $12(10.1 \%)$ & \\
\hline $\begin{array}{l}\text { Never worked/ } \\
\text { unemployed }\end{array}$ & $8(10.3 \%)$ & $19(16.0 \%)$ & \\
\hline Not known & $11(14.1 \%)$ & $18(15.1 \%)$ & \\
\hline $\begin{array}{l}\text { Maternal highest level of } \\
\text { education: }\end{array}$ & & & 0.83 \\
\hline Less than degree & $46(59.7 \%)$ & $72(62.6 \%)$ & \\
\hline Degree or higher & $20(26.0 \%)$ & $29(25.2 \%)$ & \\
\hline Not known & 11 (14.3\%) & $14(12.2 \%)$ & \\
\hline
\end{tabular}

Performance on general cognitive tests and specific mathematics tasks are shown in Table 2. Very preterm children had significantly poorer mathematical achievement than term-born controls $(t(188)=-4.24 ; P<0.001 ; d=0.62$; Figure 2a). This difference persisted after controlling for nonverbal IQ $(F(1,187)=11.65 ; P<0.001 ; d=0.61)$ and social deprivation $(F(1,185)=14.14 ; P<0.001$; Table 3$)$. Very preterm children also had significantly poorer working memory $(t(187)=-3.47 ; P<0.001 ; d=0.55)$ and visuospatial skills $(t(188)=-3.84 ; P<0.001 ; d=0.57)$, which remained significant after controlling for IMD tertile (working memory: $F(1,184)=9.80 ; P=0.002$; visuospatial skills: $F(1,185)=11.37 ; P<0.001$; Table 3 . As shown in Table 2, we did not observe significant group differences in processing speed $(t(187)=2.34 ; P=0.02)$ or inhibition $(t(188)=-2.49 ; P=0.014)$ after correction for multiple comparisons.

On tests of specific mathematics skills (Figure 2b; Table 2), very preterm children displayed significantly poorer counting proficiency than term-born controls $(t(188)=-3.21$; $P=0.002 ; d=0.48)$ and used less sophisticated strategies when solving simple arithmetic problems $(t(189)=-3.10$; 
Table 2. Children's performance on standardized achievement tests, domain general cognitive tests, and specific mathematics tests (before and after Bonferroni corrections)

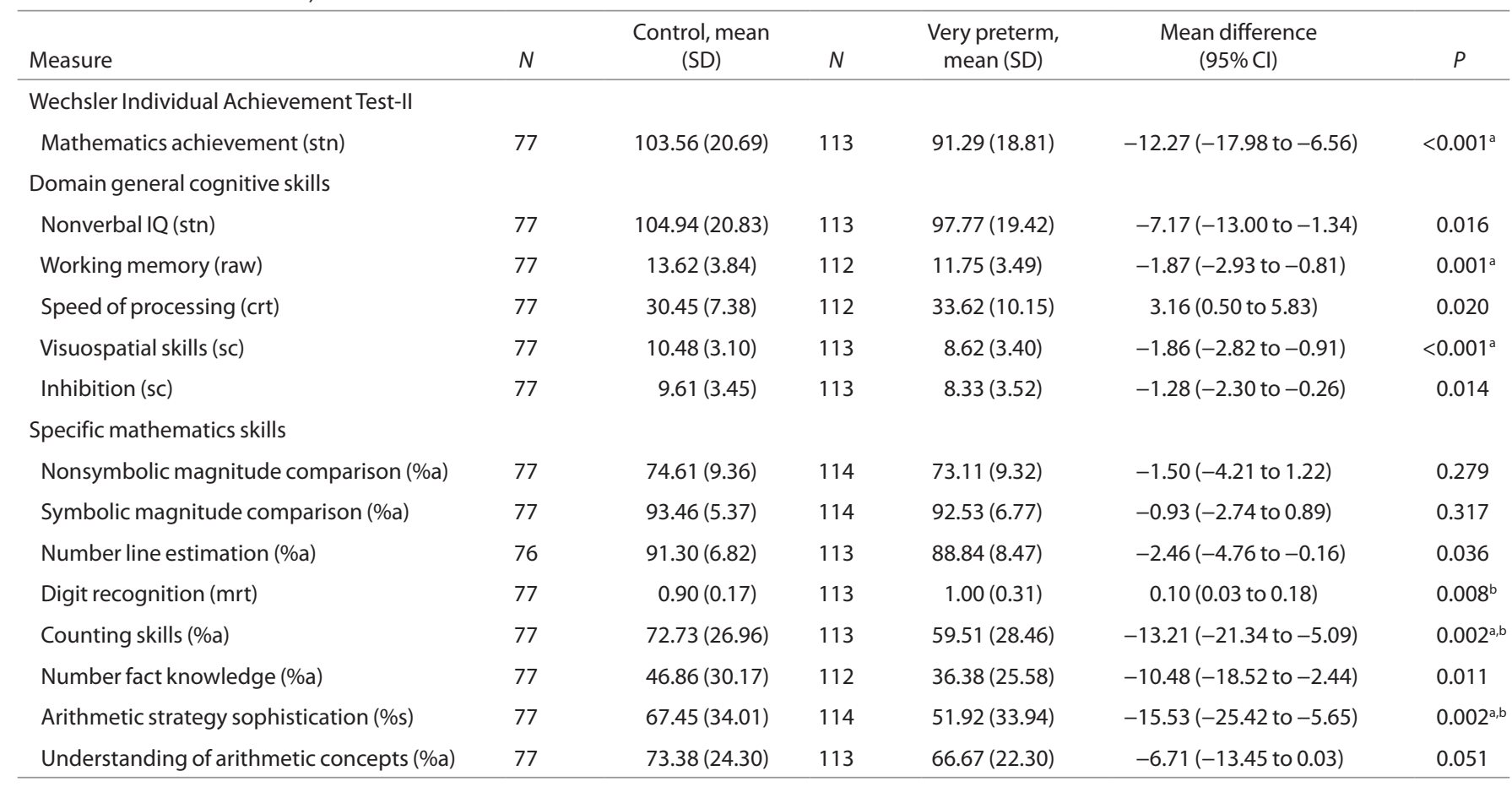

aRemains significant after applying Bonferroni correction. ${ }^{\mathrm{b} R e m a i n s ~ s i g n i f i c a n t ~ a f t e r ~ c o n t r o l l i n g ~ f o r ~ n o n v e r b a l ~ I Q . ~}$

\%a, percent accuracy score; \%s, percent use of mature strategies; crt, composite reaction time; mrt, median reaction time; raw, raw composite score for verbal and visuospatial working memory (range: 5.7-25.0); stn, standardized scores (mean: 100, SD: 15); sc, scaled scores (mean: 10, SD: 3).

$P=0.002 ; d=0.46)$; these differences persisted after controlling for social deprivation (Table 3). Importantly, group differences in strategy use and counting were negated after controlling for working memory and visuospatial skills (Table 3). There were no other significant group differences in specific mathematics tasks (Figure $2 b$ ).

Performance on the tests of basic numerical representations was assessed using accuracy and response times. All of these measures showed the same pattern of results. For the whole sample, accuracy rates were higher for the symbolic task than the nonsymbolic task $\left(M_{\text {sym }}=92.91 \% ; M_{\text {non-sym }}=73.72 \% ; F(1\right.$, $189)=645.65 ; P<0.001)$, but importantly, there was no effect of group $(F(1,189)=1.82 ; P=0.179)$ or interaction between group and task $(F(1,189)=0.14 ; P=0.705)$ on accuracy. A similar pattern was observed for response times; overall, responses were faster for the symbolic compared to the nonsymbolic version of the task $\left(M_{\text {sym }}=0.91 \mathrm{~s} ; M_{\text {non-sym }}=1.27 \mathrm{~s} ; F(1\right.$, $189)=515.58 ; P<0.001)$. Again, there was no effect of group $(F(1,189)=0.09 ; P=0.771)$ and no interaction between group and task $(F(1,189)=1.91 ; P=0.169)$.

\section{DISCUSSION}

Very preterm children had significant difficulties in mathematics and in a number of general and specific skills associated with mathematical achievement. Their deficit in mathematical achievement was of a similar magnitude to previous studies (3) and was not attributed to differences in nonverbal IQ or socioeconomic deprivation. Commensurate with previous studies, this performance was indicative of a specific problem with mathematics rather than simply reflecting a difference in general intelligence or socioeconomic factors (21). Importantly, we have shown that very preterm children did not show evidence of imprecise numerical representations and their poorer performance in specific mathematical tasks was entirely accounted for by their domain-general deficits in working memory and visuospatial skills, but not nonverbal IQ.

These results indicate that the etiology of poor mathematical achievement differs between children born very preterm and those with developmental dyscalculia. Deficits in basic numerical representations associated with developmental dyscalculia have been linked with structural and functional abnormalities in the bilateral intraparietal sulci (22). In contrast, brain development after very preterm birth has been described as an amalgam of developmental and destructive influences associated with complex neurocognitive deficits (23). Intraparietal sulcal development and function have not been studied in preterm populations, but we would predict that functional imaging during numerical representations tasks would result in different activation patterns between preterm children and those with developmental dyscalculia.

Previous studies that have attempted to establish the potential causes of preterm children's mathematical difficulties have generated conflicting results, largely due to methodological differences. In a previous study, we established a small but significant contribution of numerical representations to extremely 


\section{Articles $\mid$ Simmset al.}

a

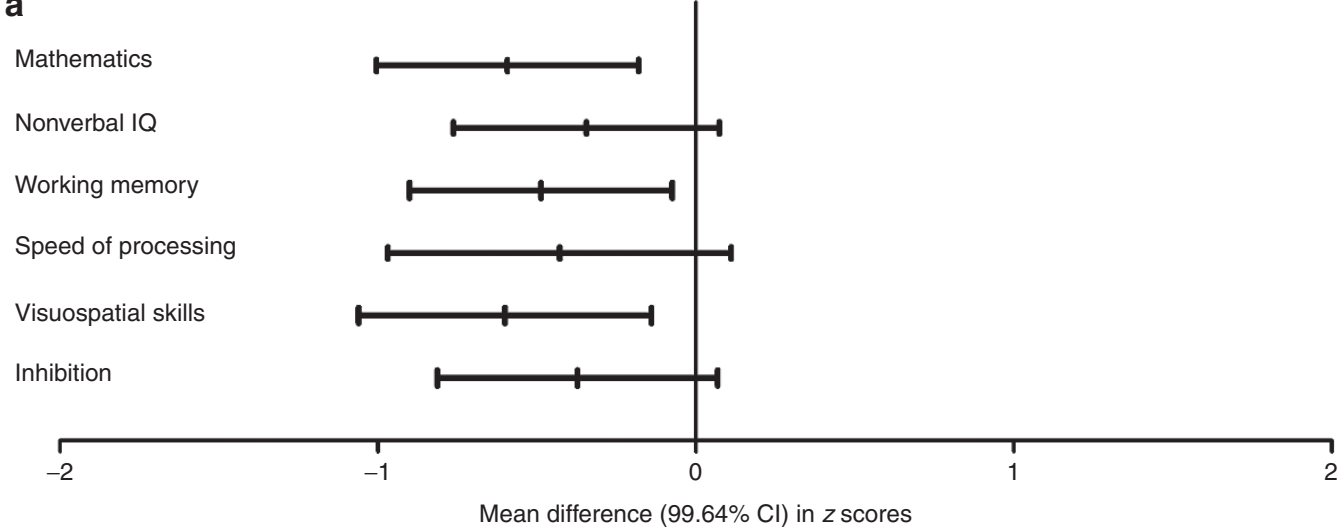

b

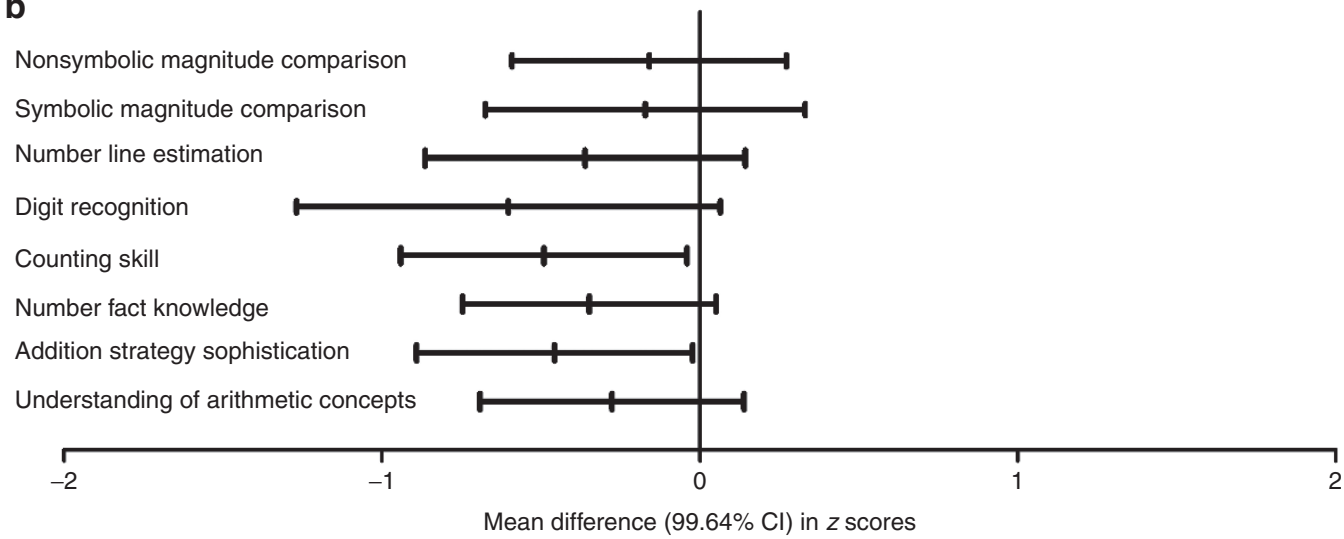

Figure 2. Mean differences ( $99.64 \%$ confidence interval) of $z$ scores between very preterm and term-born children on tasks measuring (a) domain-general cognitive skills and (b) domain-specific mathematical skills.

Table 3. Multivariate analyses of covariance results for group differences between 115 very preterm children and 77 term-born controls controlling for socioeconomic status and working memory and visuospatial skills

\begin{tabular}{|c|c|c|c|c|}
\hline Covariate & $d f$ & $F$ & $P$ & Partial $\eta^{2}$ \\
\hline \multicolumn{5}{|c|}{$\begin{array}{l}\text { Socioeconomic status (index of } \\
\text { multiple deprivation tertile) }\end{array}$} \\
\hline Mathematics & 1,185 & 14.14 & $<0.001$ & 0.07 \\
\hline Working memory & 1,184 & 9.80 & 0.002 & 0.05 \\
\hline Visuospatial skills & 1,185 & 11.37 & 0.001 & 0.06 \\
\hline Counting skill & 1,185 & 7.62 & 0.006 & 0.04 \\
\hline $\begin{array}{l}\text { Arithmetic strategy } \\
\text { sophistication }\end{array}$ & 1,186 & 7.26 & 0.008 & 0.04 \\
\hline \multicolumn{5}{|c|}{ Working memory and visuospatial skills } \\
\hline Mathematics & 1,184 & 4.41 & 0.037 & 0.02 \\
\hline Counting skill & 1,184 & 0.60 & 0.438 & 0.003 \\
\hline $\begin{array}{l}\text { Arithmetic strategy } \\
\text { sophistication }\end{array}$ & 1,184 & 1.46 & 0.229 & 0.008 \\
\hline
\end{tabular}

Effect size is reported as partial $\eta^{2}$.

preterm children's mathematical achievement, explaining $2 \%$ of the variance in performance. However, this study used a crude 12 -item measure of numerical representations in contrast to the rigorous experimental measures used in the current study.
Moreover, general cognitive factors accounted for a substantially larger proportion of the variance (70\%) than numerical representations. A recent study has also shown that mathematics difficulties in preterm children are related to deficits in IQ, rather than being specific learning difficulties, but this did not include any measures of specific mathematical skills to explore the nature of mathematics difficulties (24).

In contrast, Hellgren et al (17). recently suggested that the causes of preterm children's (unmeasured) mathematical difficulties could be attributed to imprecise numerical representations. However, we suggest that the unusual intermixed presentation of stimuli used in their study placed additional visuospatial demands on participants. In fact, in our study, we observed that very preterm children had substantial deficits in visuospatial processing and these were related to their proficiency in mathematics. This emphasizes the importance of the careful creation of experimental stimuli which take into account the known cognitive deficits of the population under study.

In the present study, we did not observe any group differences in performance using a standard and carefully controlled magnitude comparison task and thus can conclude that the measured deficits in mathematical performance in our very preterm sample were not attributable to poor numerical representations. This finding corroborates the results of Guarini et al (18). who did not find poorer numerical representations 
in their 8-y-old cohort when compared to control children. However, there is still the potential that these difficulties may be present at an earlier age and longitudinal data are required to confirm this. Although children between 8 and $10 \mathrm{y}$ old were assessed in the present study, the sample size was insufficient to assess the developmental trajectory of these skills within this cohort.

We noted that very preterm children had specific difficulties in working memory and visuospatial skills, and that these deficits accounted for group differences in strategy use and counting skills. These general cognitive skills have also been identified as being important for mathematical achievement in typically developing children $(4,7)$. Deficits in visuospatial skills and working memory are frequently reported in preterm samples (21), however, this is the first study to identify that general cognitive deficits can explain group differences in specific mathematical skills, which in turn are known to contribute to more complex mathematical performance $(4,7,18)$.

Very preterm children in the present study did not display a significant deficit in inhibition. Although this is in contrast to other studies, which have observed deficits in both inhibition and processing speed, there is some inconsistency with regard to the impact of very preterm birth on these skills (25) and, given the task dependent nature of assessment of these skills, there is variation in effect sizes dependent on the measures used (26). In particular, verbal, rather than motor, processing speed has been shown to explain very preterm children's academic deficits (27). Importantly, in the present study, we applied Bonferroni correction to adjust for multiple comparisons. Although this is a conservative approach, this minimizes the likelihood of type 1 errors and thus allows one to be sure that group differences observed in the present study are true differences. Differences between our findings and those of others may be explained by the lack of correction for multiple comparisons in previous research.

Our study has a number of clear strengths such as the unique use of a battery of both standardized and experimental tasks to elucidate the specific nature of mathematics difficulties in preterm children. The dot and digit comparison stimuli were carefully controlled to allow a rigorous assessment of numerical representations, and this is the first study to assess both general cognitive skills and specific mathematic skills alongside mathematical achievement in this population. We were also able to recruit a sample of very preterm children who were matched as closely as possible on key factors affecting academic achievement. Recruitment of control children was lower than that of very preterm children resulting in a smaller sample and a significant difference in IMD between the two groups. However, in order to ensure that matched controls were from the same class as each very preterm child this was unavoidable, and analyses were adjusted for IMD scores to account for this difference.

A priority for our research is to inform the development of interventions to improve academic performance in very preterm children. Within the English education system, all children are closely monitored and assessed by teaching staff in relation to curriculum benchmarks. Children who have identified learning disorders are provided with an Individual Education Plan, detailing specific educational learning approaches and goals for the child (28). Numerous interventions have been developed for children with mathematical (29) and working memory (30) difficulties. To date, these interventions have had varying success with different populations; however, their efficacy in improving very preterm children's educational achievement has not been established (31). Additionally, in general populations, the most frequent comorbidities with mathematical learning difficulties are dyslexia and attention deficit/hyperactivity disorders (32). In the present study, $100 \%(N=11)$ of very preterm children with standardized mathematics scores $<70$ (scores $<-2 \mathrm{SD}$ ) had identified special educational needs and $78 \%(N=31)$ of very preterm children with standardized scores $<85$ (scores $<-1$ SD) had special educational needs, compared with $16 \%$ $(N=9)$ with scores in the average range. Thus, preterm children with mathematics difficulties were clearly recognized by teachers as struggling in the classroom. Further studies are required to investigate specific comorbidities of mathematical difficulties in very preterm children, which may have implications for intervention development.

Our data suggest that rather than relying on existing interventions to enhance numerical representations developed for children with developmental dyscalculia (13), alternative interventions targeting both working memory and visuospatial abilities alongside mathematics-specific skills may be beneficial for children born preterm (33). Simply focusing on only one of these areas of deficits in interventions may have limited or null impact. For example, there has been recent interest in adaptive working memory interventions yet evidence for transfer to academic performance is lacking in children born preterm (33). Alternatively, the development of mathematics teaching methods that place fewer demands on both working memory and visuospatial skills may be beneficial; such as breaking complex tasks down into simple steps and the use of concrete manipulatives and structured worksheets to scaffold visuospatial information. These types of whole-class interventions may have wider benefits for all children in the classroom. Further research is required to develop and assess the efficacy of interventions for very preterm children.

Given the continued rise in global preterm birth rates (34) and the lack of improvement in neurocognitive outcomes for babies born at the most preterm gestations (35) identifying effective intervention strategies will have benefits not only for individual children, but society as a whole. An increasing body of evidence emphasizes the importance of early proficiency in mathematics for an individual's future employment and earning potential, in fact mathematics skills are reported as being more important than reading skills in predicting life chances (36). Improving mathematics skills in this population may therefore have far-reaching effects and may reduce the growing societal and economic burden posed by very preterm birth. 


\section{METHODS \\ Participants}

All children born $<32$ wk gestation from September 2001 to August 2003 and admitted for intensive care in two UK neonatal centers (comprising three tertiary hospitals) were invited to participate. Of 266 eligible children, the parents of 125 children responded. As the study required children to be in mainstream school and to complete psychometric tests, eight children were excluded (two lived abroad, three attended special school, and three had severe disability precluding them from participating in study tests). Therefore, 117 (44\%) were recruited to the study of whom 115 were ultimately assessed (assessments could not be scheduled for two children). For each very preterm child recruited, his/her teacher identified three classmates of the same age and sex and born at term (37-42 wk). One of these classmates was then randomly selected to participate. If consent was not received for the first child, one of the remaining children was randomly selected until a matched control participant was identified using standard procedures (37). We were unable to recruit some controls due to teachers being unable to identify a child meeting the inclusion criteria or due to lack of access to the very preterm child's school. As one control child was excluded due to prematurity, a total of 77 term-born controls were recruited.

\section{Procedure}

Ethics approval was obtained from the Derbyshire National Health Service Research Ethics Committee and informed parental consent was obtained for all children. Children were assessed in school (93\%) or at home (7\%) by one of two psychologists who were blind to group membership. Excellent inter-rater reliability was recorded with an average of $98 \%$ agreement on test items between the two psychologists. Parents were sent a feedback letter detailing their child's test results.

\section{Measures}

To assess achievement in mathematics, children completed the Wechsler Individual Achievement Test-II from which a Mathematics Composite score was derived (mean: 100; SD: 15).

General cognitive skills were assessed with standardized tests including (i) Raven's Coloured Progressive Matrices to assess nonverbal IQ from which a standardized score was derived (mean: 100; SD: 15). (ii) Three working memory tasks comprising a backwards digit recall task, a backwards word recall task, and the Mr X visuospatial working memory task from the standardized Automated Working Memory Assessment from which a composite working memory score (mean: 12.52; SD: 3.74; range: 5.67-25.00) was calculated. (iii) Rapid Automatized Naming test to assess processing speed from which an average composite score was calculated (mean: 32.33; SD: 9.23; range: 18.25-74. (iv) Developmental Neuropsychology test (NEPSY-II) arrows subtest to assess visuospatial processing and the inhibition subtest for inhibition skills; scaled scores were derived for both (mean: 10; SD: 3).

The accuracy and precision of numerical representations were measured using computerized nonsymbolic and symbolic magnitude comparison tasks (Figure 1). Two quantities were presented simultaneously on the screen and children were asked to select the more numerous. In the nonsymbolic task, quantities were sets of dots and in the symbolic task quantities were arabic digits. Presentation side of the more numerous quantity was counterbalanced. Quantities ranged from 5 to 30 and the ratio between the quantities varied $(0.5,0.6,0.7$, and 0.8 ). Dot stimuli were carefully controlled for visual characteristics of the arrays (6). Each task consisted of 80 trials and performance was assessed by accuracy and mean RT (for correct responses only). To measure skills in estimating the position of numbers on a number line, children were asked to mark the position of 22 different numbers on a series of blank number lines with the left end labeled 0 and the right 1,000 , following similar methods used in previous studies (10). A mean score for percent absolute error was calculated as the average distance between the actual and estimated positions of the numbers. This measure was reversed to indicate performance accuracy.

To explore the nature of mathematics difficulties and pin-point areas of specific deficit, mathematics skills were assessed using experimental measures adapted from Cowan et al (9). (i) In a digit recognition task, children were asked to name numbers presented randomly on a computer screen as quickly as possible. RT was recorded by a key press by the examiner and median RT to name the stimuli was calculated. (ii) Basic counting ability was measured by asking children to count aloud eight series of number sequences from memory (e.g., ascending: 2,995-3,004; descending: 325-317); overall percentage accuracy was calculated. (iii) To assess number fact knowledge, a series of 12 single-digit addition problems were read out by the experimenter and children were asked to respond with their answer as quickly as possible. Correct answers produced within $3 \mathrm{~s}$ were recorded as a known fact; the overall percent of known facts was calculated. (iv) Children completed 16 single-digit addition and subtraction problems to assess arithmetic strategy sophistication. For each problem, children were asked to give their answer and then describe how they had reached it. Children were classified as using a mature strategy if they used retrieval (recall from memory) or decomposition (mental calculation, e.g., $6+9=9+1+5$ ). The percentage of problems completed using mature strategies was recorded. (v) Finally, a series of 12 large-number addition and subtraction problems were used to assess understanding of arithmetic concepts. First, a problem with its answer was presented on the computer screen, and then beneath it a related problem was presented without an answer (e.g., $27+69=96$; 96-69=?). These problems were selected so that $8-10$-y-olds would be unlikely to be able to solve them within the specified time limit (10 s) unless they used conceptual insight, such as applying inverse or commutativity rules. The percentage of correct answers was recorded.

Parents completed questionnaire items from which three indices of socioeconomic status were derived: (i) IMD (20) tertile indicating whether the family lived in the least, middle or most deprived area of the UK; (ii) occupational class defined using National Statistics Socio-Economic Classification (38); (iii) mother's highest educational qualification dichotomized as secondary vs. higher education level.

\section{Statistical Analyses}

Independent-samples $t$-tests and $\chi^{2}$ analyses were used to assess differences in characteristics between very preterm children who were and were not recruited and between very preterm children and controls. Group differences in preterm and control children's performance on all measures were analyzed using independent samples $t$-tests. Bonferroni corrections were applied due to multiple comparisons reducing the $\alpha$-value to $P<0.0036$ (i.e., $0.05 / 14$ ). Multivariate analyses of covariance were conducted to compare performance on primary outcome measures using (i) IMD group and (ii) working memory and visuospatial skills as covariates given the group differences observed on these measures. Mixed-design ANOVA was used to investigate group differences in basic numerical representations using two metrics (accuracy and RTs).

\section{STATEMENT OF FINANCIAL SUPPORT}

This study was funded by a project grant from Action Medical Research (AMR), UK, grant number: SP4575, for the PRemature Infants' Skills in Mathematics (PRISM) Study. N.M. receives a proportion of funding from the UK Department of Health's National Institute for Health Research Biomedical Research Centres funding scheme at University College London Hospital/ University College London. C.G. is supported by a UK Royal Society Dorothy Hodgkin Fellowship.

Disclosure: All authors have no financial relationships to disclose. All authors have no conflicts of interest to disclose.

\section{REFERENCES}

1. MacKay DF, Smith GC, Dobbie R, Pell JP. Gestational age at delivery and special educational need: retrospective cohort study of 407,503 school children. PLoS Med 2010;7:e1000289.

2. Bhutta AT, Cleves MA, Casey PH, Cradock MM, Anand KJ. Cognitive and behavioral outcomes of school-aged children who were born preterm: a meta-analysis. JAMA 2002;288:728-37.

3. Simms V, Cragg L, Gilmore C, Marlow N, Johnson S. Mathematics difficulties in children born very preterm: current research and future directions. Arch Dis Child Fetal Neonatal Ed 2013;98:F457-63. 
4. Cragg L, Gilmore C. Skills underlying mathematics: the role of executive function in the development of mathematics proficiency. Trends Neurosci Educ 2014;3:63-8.

5. Raghubar KP, Barnes MA, Hecht SA. Working memory and mathematics: a review of developmental, individual difference, and cognitive approaches. Learn Individ Differ 2010;20:110-22.

6. Gilmore C, Attridge N, Clayton S, et al. Individual differences in inhibitory control, not non-verbal number acuity, correlate with mathematics achievement. PLoS One 2013;8:e67374.

7. Assel MA, Landry SH, Swank P, Smith KE, Steelman LM. Precursors to mathematical skills: Examining the roles of visual-spatial skills, executive processes, and parenting factors. Appl Dev Sci 2003;7:27-38.

8. van der Sluis S, de Jong PF, van der Leij A. Executive functioning in children, and its relations with reasoning, reading, and arithmetic. Intelligence 2007;35:427-49.

9. Cowan R, Donlan C, Shepherd DL, Cole-Fletcher R, Saxton M, Hurry J. Basic calculation proficiency and mathematics achievement in elementary school children. J Educ Psychol 2011;103:786-803.

10. Muldoon K, Towse J, Simms V, Perra O, Menzies V. A longitudinal analysis of estimation, counting skills, and mathematical ability across the first school year. Dev Psychol 2013;49:250-7.

11. Geary D, Brown S. Cognitive addition: strategy choice and speed-ofprocessing differences in gifted, normal, and mathematically disabled children. Dev Psychol 1991;27:398-406.

12. Jordan NC, Hanich LB, Kaplan D. Arithmetic fact mastery in young children: a longitudinal investigation. J Exp Child Psychol 2003;85:103-19.

13. De Smedt B, Noel MP, Gilmore C, Ansari D. The relationship between symbolic and non-symbolic numerical magnitude processing and the typical and atypical development of mathematics: evidence from brain and behavior. Trends Neurosci Educ 2013;2:48-55.

14. Mazzocco MM, Feigenson L, Halberda J. Impaired acuity of the approximate number system underlies mathematical learning disability (dyscalculia). Child Dev 2011;82:1224-37.

15. Isaacs EB, Edmonds CJ, Lucas A, Gadian DG. Calculation difficulties in children of very low birthweight: a neural correlate. Brain 2001;124(Pt 9):1701-7.

16. Guarini A, Sansavini A, Giovanelli G, et al. Basic numerical processes in preterms. World J Pediatr 2006;2:102-8.

17. Hellgren K, Halberda J, Forsman L, Adén U, Libertus M. Compromised approximate number system acuity in extremely preterm school-aged children. Dev Med Child Neurol 2013;55:1109-14.

18. Guarini A, Sansavini A, Fabbri M, Alessandroni R, Faldella G, Karmiloff-Smith A. Basic numerical processes in very preterm children: a critical transition from preschool to school age. Early Hum Dev 2014;90: 103-11.

19. Simms V, Gilmore C, Cragg L, Marlow N, Wolke D, Johnson S. Mathematics difficulties in extremely preterm children: evidence of a specific deficit in basic mathematics processing. Pediatr Res 2013;73:236-44.

20. Lad M. The English Indices of Deprivation, 2011. https://www.gov.uk/government/publications/english-indices-of-deprivation-2010.
21. Taylor HG, Espy KA, Anderson PJ. Mathematics deficiencies in children with very low birth weight or very preterm birth. Dev Disabil Res Rev 2009;15:52-9.

22. Price GR, Holloway I, Räsänen P, Vesterinen M, Ansari D. Impaired parietal magnitude processing in developmental dyscalculia. Curr Biol 2007;17:R1042-3.

23. Volpe JJ. Brain injury in premature infants: a complex amalgam of destructive and developmental disturbances. Lancet Neurol 2009;8:110-24.

24. Jaekel J, Wolke D. Preterm birth and dyscalculia. J Pediatr 2014;164: 1327-32.

25. Litt JS, Gerry Taylor H, Margevicius S, Schluchter M, Andreias L, Hack M. Academic achievement of adolescents born with extremely low birth weight. Acta Paediatr 2012;101:1240-5.

26. Aarnoudse-Moens CS, Smidts DP, Oosterlaan J, Duivenvoorden HJ, Weisglas-Kuperus N. Executive function in very preterm children at early school age. J Abnorm Child Psychol 2009;37:981-93.

27. Rose SA, Feldman JF, Jankowski JJ. Modeling a cascade of effects: the role of speed and executive functioning in preterm/full-term differences in academic achievement. Dev Sci 2011;14:1161-75.

28. Special Educational Needs: Code of Practice. London, UK: Department for Education and Skills, 2001.

29. Dowker A. What Works for Children With Mathematical Difficulties? Nottingham, UK: Department for Children, Schools and Families, 2009.

30. Melby-Lervåg M, Hulme C. Is working memory training effective? A metaanalytic review. Dev Psychol 2013;49:270-91.

31. Grunewaldt KH, Løhaugen GC, Austeng D, Brubakk AM, Skranes J. Working memory training improves cognitive function in VLBW preschoolers. Pediatrics 2013;131:e747-54.

32. von Aster MG, Shalev RS. Number development and developmental dyscalculia. Dev Med Child Neurol 2007;49:868-73.

33. Mulder H, Pitchford NJ, Marlow N. Processing speed and working memory underlie academic attainment in very preterm children. Arch Dis Child Fetal Neonatal Ed 2010;95:F267-72.

34. March of Dimes, Partnership for Maternal, Newborn and Child Health, Save the Children, World Health Organisation. Born Too Soon: The Global Action Report on Preterm Birth. Howson CP, Kinney MV, Lawn JE, eds. Geneva, Switzerland: WHO, 2012.

35. Moore T, Hennessy E, Myles J, et al. Neurological and developmental outcome in extremely preterm children born in England in 1995 and 2006: The EPICure studies. Br Med J 2012;345:e7961.

36. Crawford C, Cribb J. Reading and maths skills at age 10 and earnings in later life: A brief analysis using the British cohort study. (Report No. 3). UK: Centre for Analysis of Youth Transitions, 2013.

37. Johnson S, Wolke D, Hennessy E, Marlow N. Educational outcomes in extremely preterm children: neuropsychological correlates and predictors of attainment. Dev Neuropsychol 2011;36:74-95.

38. Office for National Statistics. The national statistics socio-economic classification user manual, 2005. http://www.ons.gov.uk/ons/guide-method/ classifications/current-standard-classifications/soc2010/soc2010-volume3-ns-sec--rebased-on-soc2010--user-manual/index.html. 